Marquette University

e-Publications@Marquette

$11-2020$

\title{
Direct Evidence of Photoinduced Charge Transport Mechanism in 2D Conductive Metal Organic Frameworks
}

\author{
James Nyakuchena \\ Marquette University \\ Sarah Ostresh \\ Yale University \\ Daniel Streater \\ Marquette University \\ Brian Pattengale \\ Marquette University \\ Jens Neu \\ Yale University
}

See next page for additional authors

Follow this and additional works at: https://epublications.marquette.edu/chem_fac

Part of the Chemistry Commons

\section{Recommended Citation}

Nyakuchena, James; Ostresh, Sarah; Streater, Daniel; Pattengale, Brian; Neu, Jens; Fiankor, Christian; Hu, Wenhui; Kinigstein, Eli Diego; Zhang, Jian; Zhang, Xiaoyi; Schmuttenmaer, Charles A.; and Huang, Jier, "Direct Evidence of Photoinduced Charge Transport Mechanism in 2D Conductive Metal Organic Frameworks" (2020). Chemistry Faculty Research and Publications. 1032.

https://epublications.marquette.edu/chem_fac/1032 


\section{Authors}

James Nyakuchena, Sarah Ostresh, Daniel Streater, Brian Pattengale, Jens Neu, Christian Fiankor, Wenhui Hu, Eli Diego Kinigstein, Jian Zhang, Xiaoyi Zhang, Charles A. Schmuttenmaer, and Jier Huang 
Marquette University

e-Publications@Marquette

\section{Chemistry Faculty Research and Publications/College of Arts and Sciences}

This paper is NOT THE PUBLISHED VERSION.

Access the published version via the link in the citation below.

Journal of the American Chemical Society, Vol. 142, No. 50 (November 2020): 21050-21058. DOI. This article is (C) American Chemical Society and permission has been granted for this version to appear in ePublications@Marquette. American Chemical Society does not grant permission for this article to be further copied/distributed or hosted elsewhere without the express permission from American Chemical Society.

\section{Direct Evidence of Photoinduced Charge Transport Mechanism in 2D Conductive Metal Organic Frameworks}

James Nyakuchena

Department of Chemistry, Marquette University, Milwaukee, Wisconsin

Sarah Ostresh

Department of Chemistry and Yale Energy Science Institute, Yale University, New Haven, Connecticut Daniel Streater

Department of Chemistry, Marquette University, Milwaukee, Wisconsin

Brian Pattengale

Department of Chemistry and Yale Energy Science Institute, Yale University, New Haven, Connecticut Jens Neu

Department of Molecular Biophysics and Biochemistry and Yale Microbial Sciences Institute, Yale University, New Haven, Connecticut

Christian Fiankor

Department of Chemistry, University of Nebraska-Lincoln, Lincoln, Nebraska 


\section{Wenhui Hu}

Department of Chemistry, Marquette University, Milwaukee, Wisconsin

\section{Eli Diego Kinigstein}

X-ray Science Division, Argonne National Laboratory, Argonne, Illinois

Jian Zhang

Department of Chemistry, University of Nebraska-Lincoln, Lincoln, Nebraska

Xiaoyi Zhang

X-ray Science Division, Argonne National Laboratory, Argonne, Illinois

\section{Charles A. Schmuttenmaer}

Department of Chemistry and Yale Energy Science Institute, Yale University, New Haven, Connecticut Jier Huang

Department of Chemistry, Marquette University, Milwaukee, Wisconsin

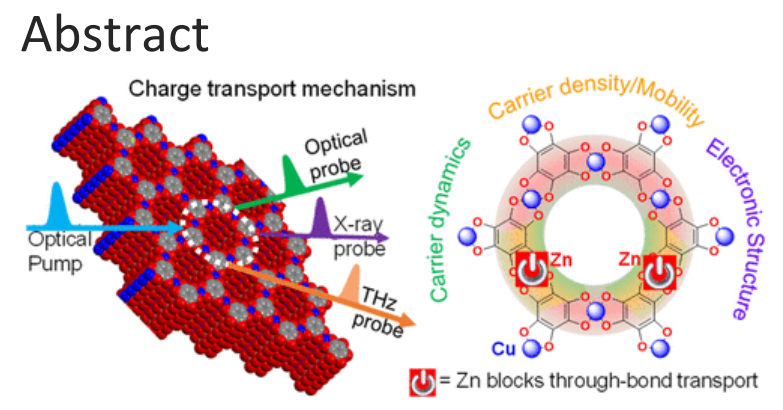

Conductive metal organic frameworks (MOFs) represent a promising class of porous crystalline materials that have demonstrated potential in photo-electronics and photocatalytic applications. However, the lack of fundamental understanding on charge transport (CT) mechanism as well as the correlation of CT mechanism with their structure hampered their further development. Herein, we report the direct evidence of CT mechanism in 2D CU-THQ MOFs and the correlation of temporal and spatial behaviors of charge carriers with their photoconductivity by combining three advanced spectroscopic methods, including time resolved optical and X-ray absorption spectroscopy and terahertz spectroscopy. In addition to Cu-THQ, the CT in Cu/Zn-THQ after incorporating $\mathrm{Zn}^{2+}$ guest metal was also examined to uncover the contribution of through space pathway, as the presence of the redox inactive $3 \mathrm{~d}^{10} \mathrm{Zn}^{2+}$ is expected to perturb the long range in-plane CT. We show that the hot carriers in $\mathrm{Cu}$-THQ generated after photoexcitation are highly mobile and undergo fast localization to a lower energy state (cool carriers) with electrons occupying Cu center and holes in ligands. The cool carriers, which have super long lifetime (>17 ns), are responsible for the long-term photoconductivity in Cu-THQ and transport through the $\mathrm{O}-\mathrm{Cu}-\mathrm{O}$ motif with negligible contribution from interlayer ligand $\pi-\pi$ stacking, as incorporation of $\mathrm{Zn}^{2+}$ in Cu-THQ significantly reduced photoconductivity. These unprecedented results not only demonstrate the capability to experimentally probe CT mechanism but also provide important insight in the rational design of 2D MOFs for photoelectronic and photocatalytic applications.

\section{Introduction}

Metal organic frameworks (MOFs) are a class of highly porous crystalline materials constructed from metal ions or clusters connected by organic ligands.(1-4) Due to their unique properties resulting from structure tunability, large surface area, and tunable porosity, MOFs have emerged as promising materials for a large variety of applications such as gas storage(5) and separation,(6) sensing,(7) catalysis,(8,9) and drug delivery.(10) In 
contrast, the use of MOFs as semiconducting light absorption and charge transport materials has received relatively little attention due to their low electrical conductivity nature.(11) The low conductivity of MOFs can be traced back to their inherent porosity, which prevents communication between adjacent units,(3) and the nature of building blocks where hard metals are often connected to redox-inactive ligands with hard base character, resulting in large energy gaps and confined electronic states.(12) Fortunately, benefiting from the abundance of building blocks and structural diversity, recent development in the field has discovered approaches to produce electrically conductive MOFs, leading to novel applications of MOFs in areas such as optoelectronics, energy storage, and artificial photosynthesis. $(8,13,14)$

Strategies that have been used to construct MOFs with charge transport (CT) properties are based on two approaches, i.e., through bond and through space approach (Scheme 1).(15) The through bond approach relies on favorable spatial and orbital overlap between metal nodes and organic linkers for effective CT. $(16,17)$ The through space approach utilizes non-covalent interactions like $\pi-\pi$ stacking between organic linkers to create an extended pathway for charge delocalization. $(18,19)$ Both approaches are expected to offer low energy pathways for charge to transport either through hopping or band transport $(15,20-22)$ and can be achieved by judicious choice of stable organic ligands and suitable combination of the metal ions and chelating units.(23) For example, 2D MOFs based on tetrathiafulvalene, naphthalene, anthracene,(24) and naphthalenediimide ligands(25) exhibit conductivity, which originates from the interlayer $\pi-\pi$ stacking between the organic ligands, allowing efficient CT from one sheet to another. MOFs based on linkers and metals that tend to form 1D second building units display charge delocalization and conductivity, which can be attributed to the intralayer CT through bonds.(26)

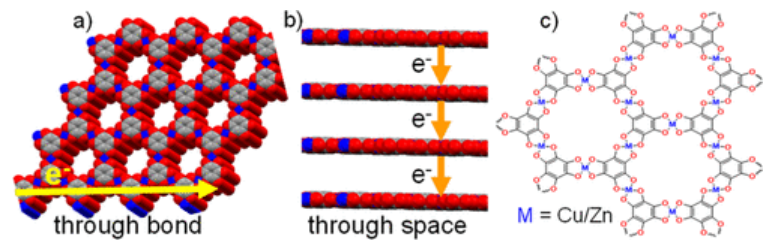

Scheme 1. Graphic Representation of $\mathrm{M}-\mathrm{THQ}(\mathrm{M}=\mathrm{Cu} \text { or } \mathrm{Zn})^{\mathrm{a}}$

${ }^{a}$ From top (a) and side view (b). The arrows in parts $a$ and $b$ illustrate through bond and through space charge transport, respectively. (c) Molecular structure of single layer M-THQ MOF.

While the field of electrically conductive MOFs has experienced tremendous expansion in the last decade and yielded a variety of MOFs with high mobility and conductivity, majority of these works focus on material design principle and conductivity measurement, leaving the fundamental understanding of CT mechanism underexplored; yet the latter is essential for the further development of this class of materials to be exploited in optoelectronics, solar cells, and photocatalysis.(27) Among the few studies that have reported CT mechanism in MOFs, methods such as probe based method $(3,28)$ and non-contact spectroscopic methods including time resolved microwave conductivity and terahertz spectroscopy have been used for measurements.(21) However, probe-based approaches lack both spatial and temporal resolution of charge carrier behaviors, resulting in ambiguity in the understanding of the CT mechanism at a molecular level. Non-contact time-resolved terahertz spectroscopy offers both temporal and spatial resolution. Still, it can only probe sufficiently mobile charges and cannot provide insight into the more localized aspects of the CT mechanism that may play a major role in the CT at a molecular level.

In response to these challenges, herein, we report the study of the CT mechanism in 2D MOFs constructed from Cu nodes connected by tetrahydroxyquinone (THQ), aiming for the direct correlation of both spatial and temporal behaviors of charge carriers with their photoconductivity by combining three advanced transient spectroscopic methods including optical transient absorption (OTA), X-ray transient absorption (XTA), and optical pump terahertz probe (OPTP) spectroscopy. In addition to $\mathrm{Cu}$-THQ, the CT in Cu/Zn-THQ after 
incorporating $\mathrm{Zn}^{2+}$ guest metal nodes was also examined to uncover the contribution of through space pathway as the presence of the redox inactive $3 d^{10} \mathrm{Zn}^{2+}$ is expected to perturb the long range in-plane charge delocalization.(29)

\section{Experimental Section}

\section{Materials}

Tetrahydroxy-1,4-benzoquinone hydrate $(96.0 \% \mathrm{TCl})$, zinc nitrate hexahydrate $\left(\mathrm{Zn}\left(\mathrm{NO}_{3}\right)_{2} \cdot 6 \mathrm{H}_{2} \mathrm{O}\right.$, Alfa Aesar), zinc acetate dihydrate $\left(\mathrm{Zn}\left(\mathrm{CH}_{3} \mathrm{COO}\right) \cdot 2 \mathrm{H}_{2} \mathrm{O}, 99 \%\right.$ Acros Organics), copper nitrate hemi pentahydrate, $\left(\mathrm{Cu}\left(\mathrm{NO}_{3}\right)_{2} \cdot 2 \cdot 5 \mathrm{H}_{2} \mathrm{O}, 99 \%\right.$ Acros Organics), ethylenediamine $\left(\mathrm{C}_{2} \mathrm{H}_{8} \mathrm{~N}_{4}, 99 \%\right.$ Acros Organics), and chloroform (Alfa Aesar, 99.8\%) were the materials used.

\section{Synthesis of M-THQ MOFs (M = Cu or Zn)}

$\mathrm{CU}$-THQ was synthesized according to previously published protocols.(30) Briefly, tetrahydroxy-1,4benzoquinone hydrate (THQ) (300 mg, $1.74 \mathrm{mmol}$ ) was dissolved in $100 \mathrm{ml}$ of degassed $\mathrm{H}_{2} \mathrm{O}$ under $\mathrm{N}_{2}$ protection. A mixture of $\mathrm{Cu}\left(\mathrm{NO}_{3}\right)_{2} \cdot 2.5 \mathrm{H}_{2} \mathrm{O}(532 \mathrm{mg}, 2.29 \mathrm{mmol})$ and ethylene diamine $(230 \mu \mathrm{L}, 3.45 \mathrm{mmol})$ in $100 \mathrm{ml}$ of degassed $\mathrm{H}_{2} \mathrm{O}$ was transferred to the THQ solution under vigorous stirring. The reaction was left to further react for $12 \mathrm{~h}$ with stirring at room temperature. The product was filtered and washed with $\mathrm{H}_{2} \mathrm{O}(100 \mathrm{ml})$ twice and acetone $(50 \mathrm{ml})$ twice. The product was collected after centrifugation at $6000 \mathrm{rpm}$ and then dried overnight in an oven at $80^{\circ} \mathrm{C}$ for further characterization. $\mathrm{Zn}^{2+}$ doping was achieved by using half the amount of $\mathrm{Cu}^{2+}(1.145$ mmol) sources with $\mathrm{Zn}\left(\mathrm{NO}_{3}\right)_{2} \cdot 6 \mathrm{H}_{2} \mathrm{O}(1.145 \mathrm{mmol})$, which resulted in the formation of mixed-node $\mathrm{Cu} / \mathrm{Zn}-\mathrm{THQ}$ MOF. To synthesize $\mathrm{Zn}-\mathrm{THQ} \mathrm{MOF}, 2.29 \mathrm{mmol} \mathrm{Zn}\left(\mathrm{NO}_{3}\right)_{2} \cdot 6 \mathrm{H}_{2} \mathrm{O}$ was used.

\section{Standard Characterization}

XRD patterns were obtained through a Rigaku Miniflex II XRD diffractometer with a Cu Ka radiation source. UVvisible absorption and diffuse reflectance data were recorded with a Cary 5000 UV-VIS-NIR spectrophotometer with an internal diffuse reflectance accessory. FTIR spectra was performed with solid samples on a Thermo Fischer Scientific iS5 FTIR spectrometer equipped with an iD3 ATR accessory. XPS measurements were performed using the Kratos AXIS Supra equipped with a monochromatic AI Ka X-ray source, running at a power of $300 \mathrm{~W}$, and operating at $15 \mathrm{kV}$. All samples were measured with a $300 \times 700 \mu \mathrm{m}^{2}$ spot size and an operating chamber pressure $<10^{-8}$ Torr.

\section{Femtosecond Optical Transient Absorption (OTA) Spectroscopy}

A regenerative amplified Ti-Sapphire laser (Solstice, $1 \mathrm{kHz}$ repetition rate, $800 \mathrm{~nm},<100 \mathrm{fs} F W H M, 3.5 \mathrm{~mJ} / \mathrm{pulse}$ ) provides the pump and probe pulses for OTA measurements. The tunable $(235-1100 \mathrm{~nm})$ pump is generated by TOPAS from $75 \%$ of the Solstice output and is chopped at $500 \mathrm{~Hz}$. The remaining $25 \%$ of Solstice output is used to generate white light in a sapphire crystal $(420-750 \mathrm{~nm})$ in a Helios ultrafast spectrometer (Ultrafast Systems LLC). The film sample on glass substrate is translated continuously to avoid sample degradation from the $450 \mathrm{~nm}$ pump pulses $(0.75 \mu \mathrm{J} /$ pulse).

\section{X-ray Transient Absorption (XTA) Spectroscopy}

The XTA experiment was performed at beamline 11-ID-D at APS and is described in our earlier report.(29) Briefly, $400 \mathrm{~nm}$ optical pump pulses were produced by a commercial regeneratively amplified Ti:Sapphire laser: a $3 \mathrm{kHz}, 100 \mathrm{fs}$ (FWHM), $3.3 \mathrm{~mJ}, 800 \mathrm{~nm}$ pulse train, which was produced by a Legend Elite Duo amplifier system seeded by a Micra-5 oscillator and pumped by an Evolution intracavity-doubled, diode pumped $\mathrm{Nd}$ :YLF Q-switched laser. The $800 \mathrm{~nm}$ signal output was then doubled with a BBO crystal to produce $400 \mathrm{~nm}$ laser pulse. The laser pump and X-ray probe are overlapped in a flowing sample stream $550 \mu \mathrm{m}$ in diameter. Avalanche photodiodes with Z-1 soller slit filters were positioned at 90 deg on both sides of the incident X-ray 
beam. The laser-on and laser-off spectra were collected at a specified delay time after the laser pump pulse excitation of a given MOF sample. The difference spectra were obtained by taking the difference between laseron and laser-off spectra.

\section{Optical Pump Terahertz Probe (OPTP) Spectroscopy}

Samples were prepared into tape cells according to the previously reported method.(31) Briefly, a business card is cut into approximately $3 \mathrm{~cm}^{2}$ pieces, a hole is punched ( $7 \mathrm{~mm}$ diameter), and acrylic tape is placed on one side of the card. The tape used is Intertape Polymer Group model number 291 (thickness $2.5 \mathrm{mil}=63.5 \mu \mathrm{m}$ ). The tape cell is cleaned with isopropanol prior to the measurement.

The measurements were taken using an amplified Ti:sapphire laser. The Spectra-Physics Spitfire Ace amplifier emits pulsed $4 \mathrm{~W}, 35 \mathrm{fs}, 800 \mathrm{~nm}$ light at $1 \mathrm{kHz}$ repetition rate. The beam is split into optical pump, terahertz generation, and detection beams. A $400 \mathrm{~nm}$ optical pump beam is generated by frequency doubling $800 \mathrm{~nm}$ light using a Type I $\beta$-barium borate (BBO) crystal (Eksma Optics). The remainder of the light is used to generate a plasma source to obtain $\mathrm{THz}$ radiation, and the detection beam is used to detect signal using free space electrooptic sampling in a ZnTe (110) crystal. Frequency-dependent experiments were performed at a pump-probe delay of $500 \mathrm{fs}$ and were processed with both no model and the thin film approximation, as described previously.(31-33) The thin film approximation yielded similar results and was used for the presented data.

\section{Time-domain Terahertz Spectroscopy (THz-TDS)}

THz-TDS was performed as described previously $(33,34)$ using an $800 \mathrm{~nm}$ oscillator (Spectra Physics Mai Tai) and photoconductive antennas for $\mathrm{THz}$ generation/detection. The measurements were performed on tape cells with an empty tape cell as a reference. A cryostat was used to collect data at 300,200 , and $100 \mathrm{~K}$ using liquid $\mathrm{N}_{2}$. Due to no apparent temperature trends, the presented data is the average over the three temperatures, two samples per material, and three locations on each sample.

\section{Computational Modeling}

Density Functional Theory (DFT) calculations were performed on user defined hexagonal unit cells each consisting of two deprotonated THQ ligands and three metal ion centers. The augmented planewave (PAW) method from the GPAW $(35,36)$ python package with Atomic Simulation Environment(37) interface was used for both structural optimization and electronic band structure calculations. The PBEsol exchange-correlation functional(38) was used for structural optimization, and the revised-PBE(39) exchange-correlation functional was used for electronic band structure calculations. Double-zeta planewave basis sets under a $400 \mathrm{eV}$ kineticenergy cutoff with a single polarization function were used for valence electrons to construct the all-electron wavefunction with frozen-core electrons. Suitable convergence was found for a $\Gamma$-centered Brillouin zone that

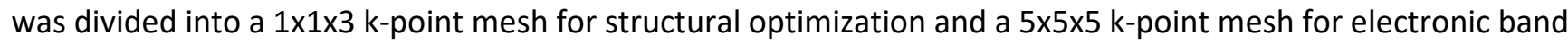
structure calculations. For structural optimization, the unit cell was allowed to optimize alongside atomic positions based on the Pulay stress estimated by the kinetic energy of an isolated atom. Convergence for the optimization was not satisfied until the forces on all atoms was less than $0.01 \mathrm{eV} / \AA$. . The electronic band structure calculations were performed on the optimized unit cells which converged to triclinic. To find a suitable path through the Brillouin zone of the triclinic unit cell along the high symmetry k-lines, an automatic detection algorithm was used.(40)

\section{Results and Discussion}

\subsection{Synthesis and Characterization of $\mathrm{M}-\mathrm{THQ}(\mathrm{M}=\mathrm{Cu}$ or $\mathrm{Zn})$}

The 2D Cu-THQ and $\mathrm{Cu} / \mathrm{Zn}-\mathrm{THQ}(\mathrm{Cu} / \mathrm{Zn}=44: 56)$ MOFs (Scheme 1$)$ are synthesized by kinetically controlled synthesis procedure following the previously published protocols (details in Experimental Section).(30) The 
Fourier transform infrared (FTIR) spectrum of the THQ ligand (Figure 1a) shows absorption features at 3350 and $1700 \mathrm{~cm}^{-1}$, which results from $\mathrm{O}-\mathrm{H}$ and $\mathrm{C}=\mathrm{O}$ stretching modes, respectively.(30) These features disappear in the $\mathrm{M}-\mathrm{THQ}$ samples, suggesting full deprotonation and reduction of the ligand due to coordination to metal nodes. Meanwhile, a new broad band at $3000 \mathrm{~cm}^{-1}$ was observed in the spectra of M-THQ MOFs. This band can be assigned to $\mathrm{NH}$ stretching modes and originated from ethylenediamine molecules within the MOF for balancing charge.(30) The formation of M-THQ is further supported by the diffuse reflectance UV-Visible-Near IR spectra. As shown in Figure 1b, THQ ligand shows a broad absorption in UV-visible region with tails extended to the near IR region corresponding to $n-\pi$ transition.(41) Compared to the spectrum of THQ ligand, an additional broad absorption was observed in the near IR region (>900 nm) of M-THQ spectra. As this broad absorption is not observed in the spectrum of Zn-THQ (Figure S1), we assigned this absorption to either the intramolecular charge transfer band or Cu d-d transition, in good agreement with previous data of Cu-THQ.(30)

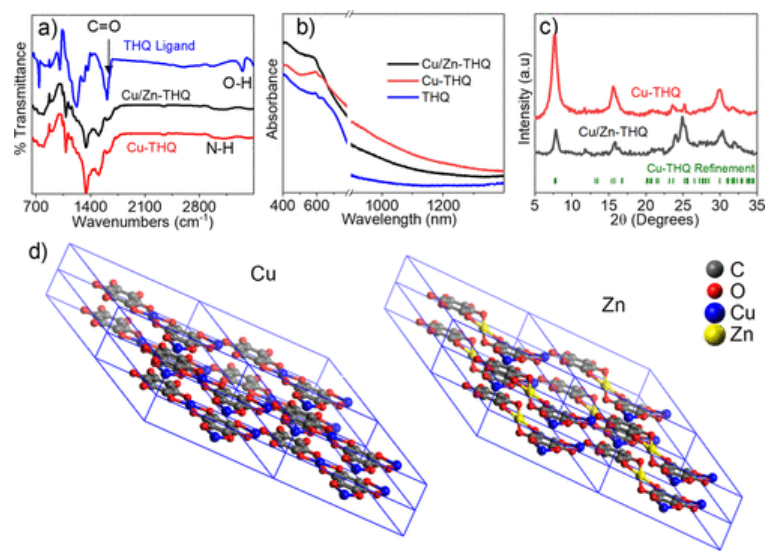

Figure 1. FTIR (a) and diffuse reflectance spectra (b) of Cu-THQ, Cu/Zn-THQ, and THQ ligands in solid state. (c) The XRD patterns of $\mathrm{Cu}-\mathrm{THQ}$ and $\mathrm{Cu} / \mathrm{Zn}-\mathrm{THQ}$. (d) $\mathrm{Cu}$ and $\mathrm{Zn}$ local coordination geometry in M-THQ MOF.

Powder X-ray diffraction (XRD) was used to examine the crystalline nature of M-THQ. As shown in Figure 1c, the XRD patterns of M-THQ agree well with the standard Kagome lattice and previously published patterns for $\mathrm{Cu}$ THQ,(30) further supporting the formation of crystalline M-THQ. Rietveld refinement of Cu-THQ (green lines in Figure 1c) suggest that it belongs to the $\mathrm{Cmcm}$ space group with a base-centered orthorhombic unit cell in line with previous works.(30) Density functional theory (DFT) calculations with PBEsol exchange-correlation functional predicts that $\mathrm{CU}$-THQ will arrange itself in a planar manner within a triclinic unit cell that is nearly hexagonal (Figure 1d, left panel). The planar arrangement occurs owing to the preferential square planar arrangement of the $\mathrm{Cu}^{2+}$ ion. The $\mathrm{Zn}^{2+}$ variant is distorted arising from $\mathrm{Zn}^{2+}$ preferentially adopting a coordination environment that is influenced by $\mathrm{O}^{1-}$ ions in adjacent layers (Figure $1 \mathrm{~d}$, right panel). The $\mathrm{N}_{2}$ adsorption isotherm (Figure S2) at $77 \mathrm{~K}$ reveals a BET surface area of $139.08 \mathrm{~m}^{2} / \mathrm{g}$ for $\mathrm{Cu}$-THQ in agreement with earlier reports.(30) A similar result was obtained for $\mathrm{Cu} / \mathrm{Zn}-\mathrm{THQ}\left(159.19 \mathrm{~m}^{2} / \mathrm{g}\right)$, showing maintenance of the intrinsic porosity on $\mathrm{Zn}$ dilution.

X-ray photoelectron spectroscopy was used to determine the charges on M-THQ MOFs. The XPS scan of $\mathrm{Cu}_{2} \mathrm{P}_{3 / 2}$ in both $\mathrm{Cu}-\mathrm{THQ}$ and $\mathrm{Cu} / \mathrm{Zn}-\mathrm{THQ}$ displays a dominant peak at $933 \mathrm{eV}$, satellite peaks characteristics of $\mathrm{Cu}^{2+}$, and a trace amount of $\mathrm{Cu}^{+}$(Figure S3a), which agree with the previous literature data,(30) suggesting the presence of a mixed valence state of $\mathrm{Cu}$ center in both samples. Furthermore, $\mathrm{O}$ 1s XPS scans (Figure S3b) shows that the binding energy for the $\mathrm{O}$ 1s electrons increases in the order of $\mathrm{Cu}-\mathrm{THQ}, \mathrm{Cu} / \mathrm{Zn}-\mathrm{THQ}$, and $\mathrm{Zn}-\mathrm{THQ}$, suggesting that electron density on the O-M-O motif reduces with $\mathrm{Zn}$ dilution. 


\subsection{CT Mechanism by Probing Exciton Dynamics using OTA}

Femtosecond optical transient absorption (OTA) spectroscopy was used to examine the carrier dynamics and CT mechanism in Cu-MOF and Cu/Zn-MOF. Figure 2a shows the OTA spectra of Cu-THQ following $450 \mathrm{~nm}$ excitation. Immediately following the excitation, the OTA spectra show a broad negative feature at 470-660 nm and a positive feature at $>700 \mathrm{~nm}$ (denoted as E1). Recent computational studies(42) suggested that Cu-THQ behaves as a semiconductor with a highly dispersive conduction band, suggesting that a semiconductor exciton model rather than a molecular model should be used to interpret OTA spectra. As a result, the negative feature can be assigned to the exciton bleach (EB) band resulting from the reduced oscillation strength of the transition from the valence band (VB) to the conduction band (CB) due to electrons filling the $C B .(43,44)$ The positive feature (E1) can be attributed to the electron absorption due to the transition from the lower to the higher energy level of $\mathrm{CB}$. The EB band centered at $620 \mathrm{~nm}$ recovers, and E1 decays quickly with time, where an isosbestic point was observed at $700 \mathrm{~nm}$, suggesting that they represent the same relaxation process, i.e., electrons in the $\mathrm{CB}$ recombine with the hole in VB. It is interesting to note that the $\mathrm{EB}$ at $\sim 525 \mathrm{~nm}$ evolves to be positive after $5 \mathrm{ps}$, indicating that $\mathrm{EB}$ at $\sim 525 \mathrm{~nm}$ overlaps with electron absorption at the same region (denoted as E2), where E2 has a different nature from E1 as E2 decays slower than E1 at early time. These spectral evolutions can be more clearly seen from the comparison of their kinetic traces (Figure 2b), where the kinetics of EB $(620 \mathrm{~nm})$ follow E1 $(700 \mathrm{~nm})$ but E2 $(525 \mathrm{~nm})$ becomes positive at later time.
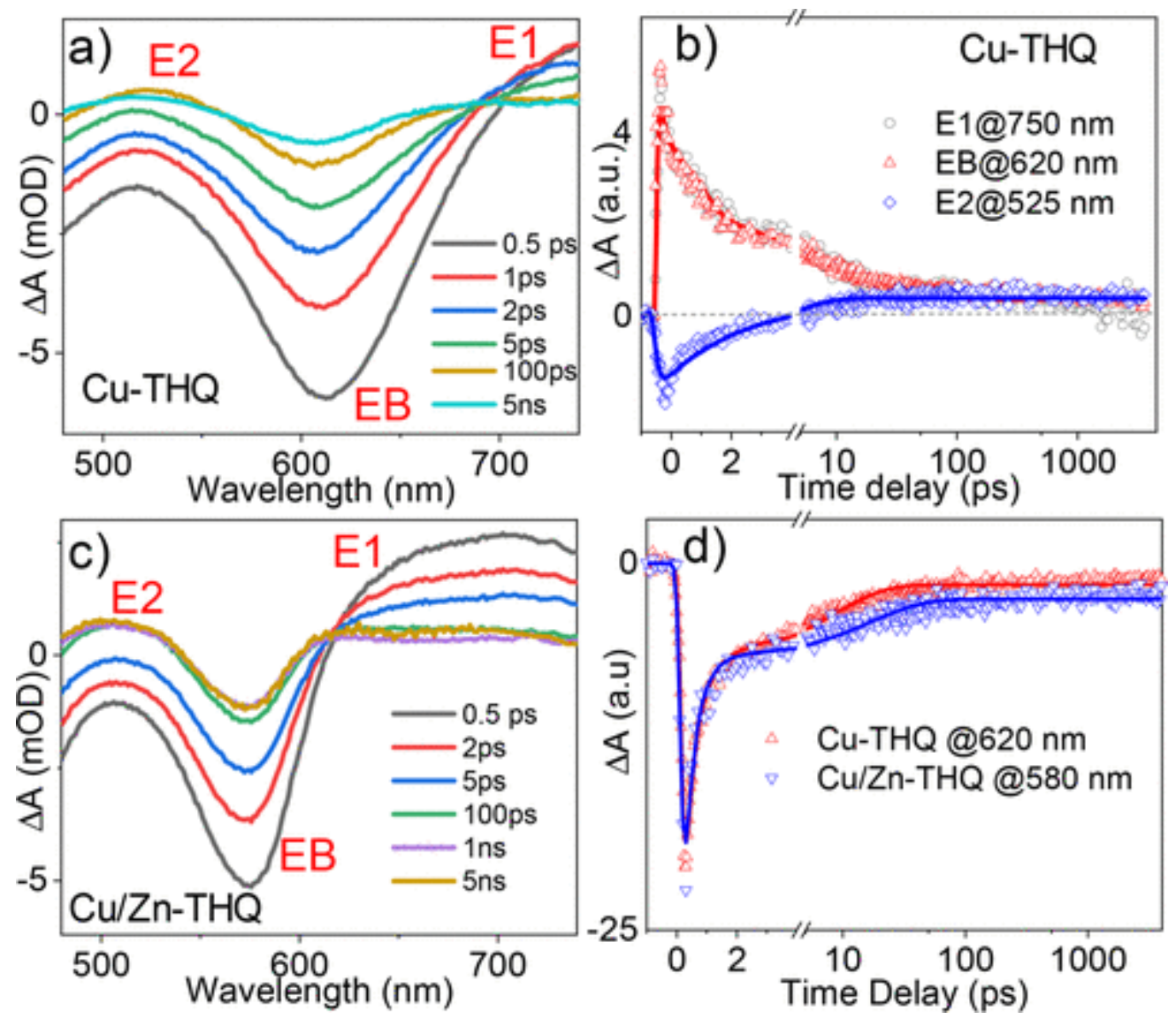

Figure 2. OTA spectra of Cu-THQ (a) and Cu/Zn-THQ (c). (b) The comparison of kinetic traces of Cu-THQ at 750, 620, and $525 \mathrm{~nm}$. (d) The comparison of kinetic traces of EB band for Cu-THQ at $620 \mathrm{~nm}$ and Cu/Zn-THQ at 580 $\mathrm{nm}$.

As the OTA spectra of the THQ ligand in chloroform solution (Figure S4a) show distinct features, i.e., ground state bleach centered at $480 \mathrm{~nm}$ and excited state absorption with well-defined band centered at $560 \mathrm{~nm}$, the 
conjugated structure in Cu-THQ must be responsible for the observed spectral features. In addition, the OTA spectra of Zn-THQ (Figure S4b) also show very distinct features from that of Cu-THQ, where a much sharper negative band at $570 \mathrm{~nm}$ and two well-defined positive features centered at 520 and $625 \mathrm{~nm}$ were observed in the spectra of $\mathrm{Zn}-\mathrm{THQ}$. Given that the $\mathrm{Zn}^{2+}$ node with closed shell $3 \mathrm{~d}^{10}$ electron configuration is anticipated to prevent the ligand-M-ligand through-bond CT pathway in Zn-THQ,(29) the spectral evolutions in the OTA spectra of $\mathrm{Cu}$-THQ are likely due to the delocalized intralayer excitons which transport via through-bond pathway. The OTA spectra of Cu/Zn-THQ (Figure 2c) show similar spectral shape (EB, E1, and E2) as that of Cu-THQ. However, compared to that of $\mathrm{Cu}-\mathrm{THQ}$, the EB band in $\mathrm{Cu} / \mathrm{Zn}-\mathrm{THQ}$ is narrower. In addition, significant blue shift was observed for EB and E1 with respect to $\mathrm{CU}$-THQ, yet a negligible shift was observed for E2. Previous studies have shown that the broadness of the OTA spectral features in a zirconium based MOF (NU-1000) is associated with the presence of loosely bound excitons and high carrier density.(45) As a result, the broader EB in Cu-THQ than that in $\mathrm{Cu} / \mathrm{Zn}-\mathrm{THQ}$ suggests the decreasing delocalization of carriers and carrier density in M-THQ when some $\mathrm{Cu}^{2+}$ ions are replaced by $\mathrm{Zn}^{2+}$, which is consistent with photoconductivity measurements discussed later. This also explains the hypsochromic shift of EB in the spectra of $\mathrm{Cu} / \mathrm{Zn}$-THQ with respect to $\mathrm{Cu}$-THQ, where $\mathrm{Zn}^{2+}$ partially blocks intralayer charge delocalization, resulting in more tightly bound excitons with lower mobility and/or carrier density and thus fewer dispersive states.

Note that E2 was observed in the spectra of both samples and shows negligible shift upon the incorporation of $\mathrm{Zn}^{2+}$, indicating that the degree of intralayer delocalization through ligand-Cu-ligand conjugated motifs has little impact on E2. This may suggest that E2 originates from localized ligand states and/or interlayer excitons as a result of ligand $\pi-\pi$ conjugation. We can exclude the contribution of interlayer CT (through space) to E2 as negligible photoconductivity was observed in Zn-THQ (Figure S5). As a result, we attribute E2 to the absorption due to localized ligand state, i.e., tightly bound excitons. This also explains the slower decay kinetics of E2 than $\mathrm{E} 1$ at early time in $\mathrm{Cu}-\mathrm{THQ}$ and $\mathrm{Cu} / \mathrm{Zn}-\mathrm{THQ}$, as loosely bound intralayer excitons (E1) may undergo additional ultrafast localization process to form localized excitons. This assignment is further supported from the comparison of their kinetic traces of EB (Figure 2d), which shows that EB recovery in Cu-THQ is faster than in $\mathrm{Cu} / \mathrm{Zn}$-THQ. The OTA kinetic traces can be fit by a three-component decay function. The best fits to the kinetics are also shown in Figure $2 d$ with their fitting parameters listed in Table S1. The ultrafast time constant $\left(\tau_{1} \sim 0.4\right.$ ps) in both samples may result from exciton localization to ligand state or trapping process, while $\tau_{2}(\sim 8-17$ ps) and $\tau_{3}(\gg 5 \mathrm{~ns})$ may be attributed to exciton recombination time constants. $\tau_{3}$ is much longer than our OTA time window ( $5 \mathrm{~ns})$, suggesting that M-THQ has long-lived intralayer excitons.

\subsection{Unravelling the Atomic-level Nature of Long-lived Intralayer Exciton using XTA}

To gain insight into the nature of the long-lived intralayer excitons, we measured the electronic structure at the Cu center in both Cu-THQ and Cu/Zn-THQ using X-ray transient absorption (XTA) spectroscopy, a powerful technique that is element sensitive and allows us to directly probe the electron density at the Cu site upon photoexcitation with a $400 \mathrm{~nm}$ laser pump pulse.(46)Figure 3a shows the ground state X-ray absorption near edge structure (XANES) spectrum of CU-THQ as well as its XANES difference spectra collected at 100 ps and 5 ns after excitation. The difference spectra were obtained after subtracting the laser-off (ground state) spectrum from the laser-on (with excitation) spectrum. Two prominent positive features observed at 8.983 and $8.989 \mathrm{keV}$ in the difference spectrum of $\mathrm{Cu}-\mathrm{THQ}$, assigned to $1 \mathrm{~s} \rightarrow 4 \mathrm{p}_{z}$ (+ shakedown) and the main $1 \mathrm{~s} \rightarrow 4 \mathrm{p}_{x y}$ transitions, respectively, $(47,48)$ indicate that the absorption edge moves to lower energy, i.e., the $\mathrm{Cu}$ center in $\mathrm{Cu}$-THQ is reduced upon photoexcitation.(49) Because $\mathrm{Cu}$ and THQ ligand are the only two components in $\mathrm{Cu}$-THQ, the reduction of $\mathrm{Cu}$ center must be accompanied by the oxidation of THQ, suggesting that the nature of the intralayer excitons has electrons located at the $\mathrm{Cu}$ center while holes are located at THQ. The splitting of the 1s$4 p$ transition into $1 s \rightarrow 4 p_{z}$ and $1 s \rightarrow 4 p_{x y}$ transitions likely results from the square planar geometry of Cu center in $\mathrm{Cu}$-THQ, which has no ligand orbitals in the $z$-direction and thus lowers the energy of the $1 s-4 p_{z}$ transition. 

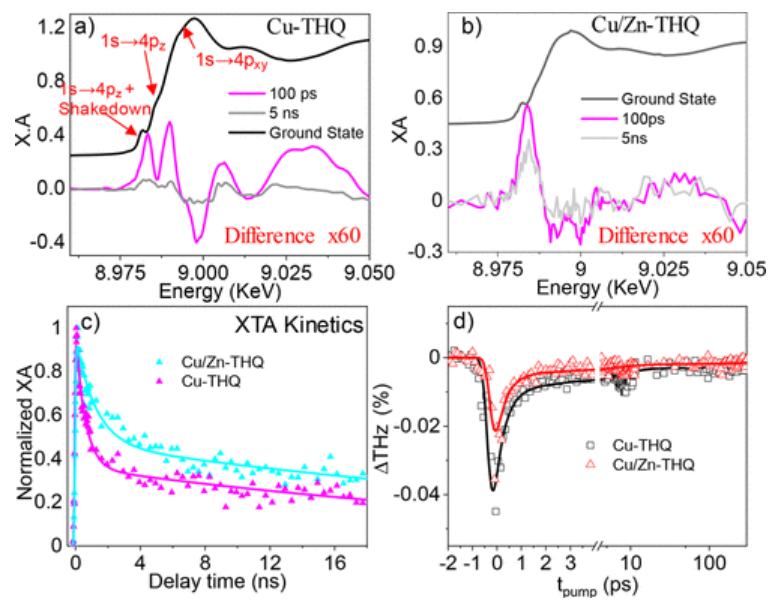

Figure 3. Difference XANES spectra of Cu-THQ (a) and Cu/Zn-THQ (b), which were obtained by subtracting the laser-off (ground state) spectrum (black plot) from laser-on spectrum collected at $100 \mathrm{ps}$ and $5 \mathrm{~ns}$. (c) The comparison of XTA kinetics for Cu-THQ at $8.989 \mathrm{keV}$ and Cu/Zn-THQ at $8.983 \mathrm{keV}$. (d) The comparison of OPTP traces of the $\mathrm{Cu}-\mathrm{THQ}$ and $\mathrm{Cu} / \mathrm{Zn}-\mathrm{THQ}$.

However, the $1 \mathrm{~s} \rightarrow 4 \mathrm{p}_{x y}$ transition is no longer prominent in Cu/Zn-THQ (Figure $3 \mathrm{~b}$ ) and only the 1s$4 p_{z}$ transition with shakedown contribution at $8.983 \mathrm{keV}$ is observed. Because the presence of the double transition of $\mathrm{CU} \mathrm{K}$ edge XANES spectrum in $\mathrm{Cu}(\mathrm{II})$ bis-diimine complexes was believed to be associated with high degree of covalency, $(49,50)$ the absence of the $1 s \rightarrow 4 p_{x y}$ transition in Cu/Zn-THQ suggests less covalency in $\mathrm{Cu} / \mathrm{Zn}$-THQ with respect to that in Cu-THQ. This is because the introduction of $\mathrm{Zn}^{2+}$ into $\mathrm{Cu}$-THQ prevents intralayer charge delocalization, which reduces the degree of in plane covalency. These results together suggest that the intralayer excitons are transported through ligand-Cu-ligand pathway, where the presence of $\mathrm{Zn}$ ion in $\mathrm{M}$-THQ reduces in plane covalency and blocks through-bond transport, which is consistent with our OTA results and OPTP results discussed later. The consistency of the XTA and OTA results was further supported from the comparison of XTA kinetic traces of Cu-THQ (probed at $8.983 \mathrm{keV}$ ) and Cu/Zn-THQ (probed at $8.989 \mathrm{keV}$ ). As shown in Figure $3 c$, the kinetics of both samples show a super long-lived component ( $>17 \mathrm{~ns}$ ), corresponding to the long time constant $\left(\tau_{3}\right)$ in OTA kinetics. Similar to OTA results, the kinetic trace of Cu-THQ decays much faster than that in $\mathrm{Cu} / \mathrm{Zn}-\mathrm{THQ}$, suggesting shorter lived excitons in the former than the latter.

\subsection{Photoconductivity Dynamics via OPTP Spectroscopy}

The OTA and XTA results together suggest that photoexcitation of CU-THQ leads to the formation of long-lived intralayer charge carriers, comprising electrons and holes, with electrons being mainly Cu-localized and holes being mainly THQ ligand-localized. Moreover, it was found that the introduction of $\mathrm{Zn}^{2+}$ to M-THQ partially blocks intralayer charge carrier delocalization. The natural question following these interesting findings is whether these delocalized intralayer charge carriers exhibit photoconductivity. In order to uncover the correlation of intralayer charge carriers and photoconductivity behavior of M-THQ, we measured the photoconductivity of these samples by probing the free carrier dynamics using optical pump terahertz probe (OPTP) spectroscopy.(21,32,51-55)Figure 3d shows OPTP dynamics for Cu-THQ and Cu/Zn-THQ following 400 $\mathrm{nm}$ excitation. Note that both samples are adequately thick to fully absorb the pump pulse. It can be seen that photoconductivity is present in both samples evidenced by the attenuation of THz after photoexcitation. At time zero (a pump delay $t_{\text {pump }}=0$ ), a rapidly decaying peak in the signal is observed, which is followed by a slower decay at longer delay. The normalized traces (Figure S5) show that the dynamics observed are very similar in both samples and that the traces do not decay to baseline within the measured 300 ps pump-probe delay time window, suggesting a long-lived component of the photoconductivity. It is interesting to note that the inclusion of $\mathrm{Zn}$ into $\mathrm{Cu}$-THQ results in an approximately $50 \%$ decrease of the peak OPTP signal. Supporting this trend, it 
was observed that the photoconductivity of Zn-THQ is below the detection limit of OPTP experiments (Figure S5). Because the measured photoconductivity is a product of the carrier mobility and carrier density,(56) we can conclude that incorporation of $\mathrm{Zn}$ into MOFs significantly reduces the carrier mobility and/or density, consistent with OTA and XTA results. These qualitative observations are based on the peak $\mathrm{THz}$ attenuation within the IRF time scale; however, modeling demonstrates the same trend, as discussed below.

Because similar decay dynamics were observed for both MOFs, the same multiexponential fit model was used to model the photoconductivity dynamics.

$$
\Delta T H z(t)=-\left(A_{1}\left(e^{-\left(t-t_{0}\right) / \tau_{1}}\right)+A_{2}\left(e^{-\left(t-t_{0}\right) / \tau_{2}}\right)+A_{3}\left(e^{-\left(t-t_{0}\right) / \tau_{3}}\right)\right) \otimes G_{R}
$$

Three-time components, represented as the time constants $\tau_{1}, \tau_{2}$, and $\tau_{3}$, were required to adequately describe the decay dynamics, and therefore they were fit to a gaussian-convoluted triexponential function (eq 1) with best fit parameters shown in Table S3. The first-time component $\left(\tau_{1}\right)$ is instrument response-limited and immediately follows the incident pump pulse. It is fixed to $0.4 \mathrm{ps}$, which is equivalent to the FWHM of the gaussian instrument response function (IRF) that is convoluted with the fit model. The decay of the trace appears to have 2 additional time components, one on the time scale of 2-5 ps $\left(\tau_{2}\right)$ and one that acts as a baseline offset $\left(\tau_{3}\right)$ to describe remaining photoconductivity signal persistent beyond the measured delay range of 300 ps. The decay in THz attenuation is due to the loss of mobile carriers. This loss can be attributed to a variety of physical processes, such as electron-hole recombination, carrier trapping to non-mobile states, and relaxation to lower energy states with lower mobility. $(56,57)$ The ground state refractive indices of $\mathrm{Cu}-\mathrm{THQ}$ and $\mathrm{Cu} / \mathrm{Zn}$-THQ were determined using time-domain terahertz spectroscopy (THz-TDS) and are shown in Figure S6ac. These optical properties are within uncertainty between $\mathrm{Cu}-\mathrm{THQ}$ and $\mathrm{Cu} / \mathrm{Zn}-\mathrm{THQ}$, suggesting similar ground state properties in the $\mathrm{THz}$ frequency regime. The frequency-dependent photoconductivity of $\mathrm{Cu}$-THQ was measured at a time delay ( $t_{\text {pump }}$ ) of $500 \mathrm{fs}$, which is in the time regime of $\tau_{1}$ and $\tau_{2}$. The photoconductivity (Figure $\mathrm{S} 6 \mathrm{~d}$ ) shows a value of approximately $0.2 \mathrm{~S} / \mathrm{m}$. While the signal for $\mathrm{Cu} / \mathrm{Zn}-\mathrm{THQ}$ was prohibitively small to measure in frequency-dependent experiments, the OPTP results in Figure $3 \mathrm{~d}$ suggest that the photoconductivity of $\mathrm{Cu} / \mathrm{Zn}$ THQ is approximately half of that of $\mathrm{Cu}$-THQ (i.e., $0.1 \mathrm{~S} / \mathrm{m}$ ).

With a combination of the results from OTA, OPTP, and XTA studies, $\tau_{1}$ may result from carrier trapping or relaxation to lower energy states with lower mobility (e.g., partial localization) while $\tau_{2}$ and $\tau_{3}$ represent a decay component that is beyond the typical time scale for carrier trapping and can be assigned to the recombination of electrons and holes. The presence of long-lived time constant $\left(\tau_{3}\right)$ in OPTP experiment suggests that the longlived intralayer excitons observed in OTA and XTA experiments are photoconductive and are featured by $\mathrm{Cu}$ localized electrons and THQ ligand-localized holes.

\subsection{Electronic Structure Predicted by DFT and the Correlation with Spectroscopic Results}

To gain even more insight on the nature of these photoconductive intralayer excitons, we performed electronic structure DFT calculations with revised PBE exchange-correlation functional. The computational methodology predicted remarkably different VB and CB for Zn-THQ and Cu-THQ (Figure 4a,b). For each of the MOFs, the VB crosses the respective Fermi level (FL), which indicates that ground state conductivity is thermodynamically possible. The distinct difference arises from the dispersion of the FL crossing VB and the density of states (DOS) present both at and above the FL. The VB of Zn-THQ crosses the FL only marginally along the $X-\Gamma k$-line. Along the $Y-\Gamma$ and $Z-\Gamma$ k-lines, the VBs have low dispersity $\left(9.1 m_{e}\right.$ and $27 m_{e}$, respectively). In contrast, most of the VB of $\mathrm{Cu}$-THQ resides above the FL and has more dispersion than that in Zn-THQ, which can be best illustrated by a 
20\% lower effective electron mass along the $\mathrm{X}-\Gamma \mathrm{k}$-line in $\mathrm{Cu}$-THQ than $\mathrm{Zn}$-THQ. In addition, the dispersions along the $\mathrm{Y}-\Gamma\left(4.7 \mathrm{~m}_{\mathrm{e}}\right)$ and Z- $\Gamma\left(3.5 \mathrm{~m}_{\mathrm{e}}\right) \mathrm{k}$-lines as well as the DOS at the FL in Cu-THQ are also much higher than that in $\mathrm{Zn}-\mathrm{THQ}$. Moreover, the atom resolved DOS reveals that the VB of Zn-THQ is not primarily comprised of orbitals belonging to the $\mathrm{Zn}^{2+}$ ion, which suggests that any carrier mobility must proceed through the organic portions of the framework. This is further reflected in the bands above the $\mathrm{FL}$, which also results predominantly from electron orbitals of carbon atoms. In contrast, the predominant contribution to the VB in Cu-THQ comes from Cu and $\mathrm{O}$ orbitals and the bands above the FL have large DOS values that primarily arise from the electron orbitals of $\mathrm{Cu}$. These results together suggest the covalency in the $\mathrm{O}-\mathrm{Cu}-\mathrm{O}$ bond, through which ground and excited state carrier transport can evidently proceed, and the incorporation of $\mathrm{Zn}^{2+}$ in $\mathrm{Cu}-\mathrm{THQ}$ is anticipated to reduce carrier density and/or mobility. These computational results are consistent with our spectroscopic data, where the greater EB band broadening observed in the OTA spectra of Cu-THQ than that in Cu/Zn-THQ indicates the presence of more delocalized carriers which are transported through the $\mathrm{O}-\mathrm{Cu}-\mathrm{O}$ bond (XTA results), consistent with higher photoconductivity (larger OPTP signal) in Cu-THQ than that in Cu/Zn-THQ.
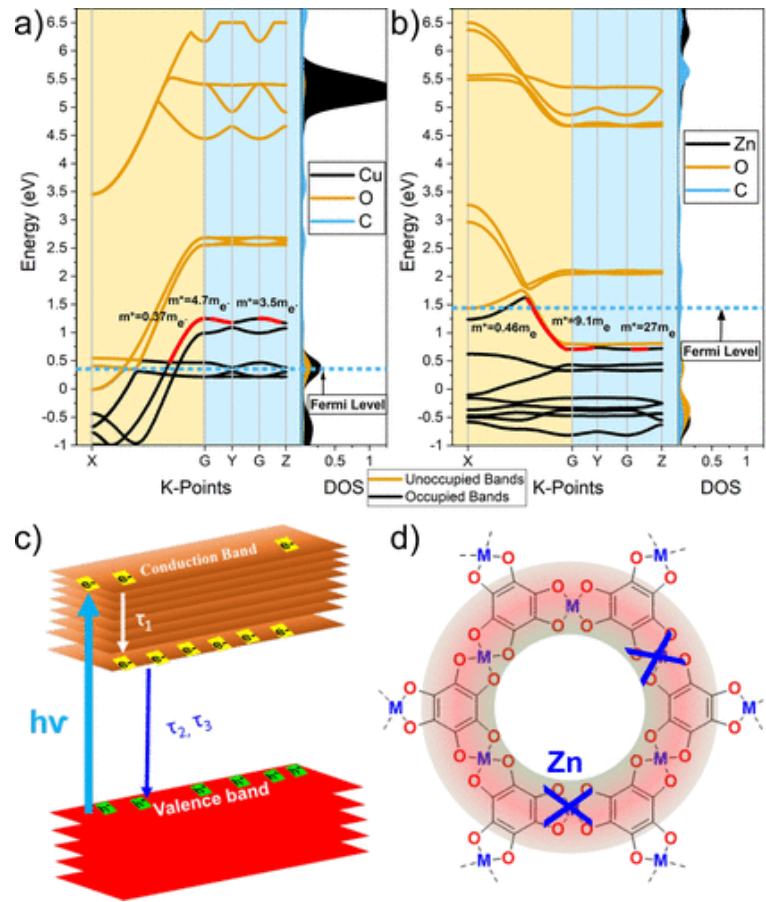

Figure 4. Band structure diagrams (see Figure S10 for real space interpretation of Brillouin zone) and atomic orbital projected density of states calculated using the revised PBE exchange-correlation functional for Cu-THQ (a) and Zn-THQ (b). (c) Schematic representation of exciton relaxation dynamics. (d) The cartoon illustrating that the incorporation of $\mathrm{Zn}^{2+}$ to $\mathrm{Cu}-\mathrm{THQ}$ blocks intralayer exciton delocalization.

Taking the spectroscopic and computational results together, we proposed a model for the CT mechanism in CuTHQ. As shown in Figure 4c, the photoexcitation of $\mathrm{Cu}$-THQ promotes electrons from the $\mathrm{VB}$ to $\mathrm{CB}$, generating hot electrons in $\mathrm{CB}$ and holes in the VB. These hot carriers have high mobility and quickly relax down to a lower energy level in CB/VB that has smaller mobility (more localized state) as supported by both OTA and OPTP. This relaxation process corresponds to the ultrafast decay time $\left(\tau_{1}\right)$ observed in both OTA and OPTP. The electron and hole recombination then occur with time constants ranging from a few picoseconds $\left(\tau_{2}\right)$ to $\gg 5 \mathrm{~ns}\left(\tau_{3}\right)$. OPTP results indicate that the short-lived hot and long-lived cool carriers in $\mathrm{Cu}$-THQ all exhibit mobility, suggesting that $\mathrm{Cu}$-THQ is photoconductive. The photoconductivity of $\mathrm{Cu}-\mathrm{THQ}$ is reduced by half upon replacing $\sim 50 \%$ of $\mathrm{Cu}^{2+}\left(3 \mathrm{~d}^{9}\right)$ by $\mathrm{Zn}^{2+}\left(3 \mathrm{~d}^{10}\right)$ and disappears in $\mathrm{Zn}$-THQ, suggesting that the intralayer rather than interlayer transport is responsible for the observed photoconductivity (Figure 4d). As suggested by both XTA and DFT results, the 
photoconductivity of $\mathrm{Cu}-\mathrm{THQ}$ primarily proceeds through the $\mathrm{O}-\mathrm{Cu}-\mathrm{O}$ motif with negligible contribution from interlayer $\pi-\pi$ stacking between the THQ ligands.

\section{Conclusion}

In summary, we report direct evidence of the charge transport mechanism in Cu-THQ MOF and the correlation of temporal (sub-picosecond to nanosecond regime) and spatial (atomic level) behaviors of carriers with their photoconductivity using the combination of three advanced time resolved spectroscopies. We show that the hot carriers (high mobility) generated after photoexcitation undergo an ultrafast localization process and relax down to a lower energy state (cool carriers) with super long lifetimes (>17 ns). The long-lived cool carriers are transported through the $\mathrm{O}-\mathrm{Cu}-\mathrm{O}$ motif with negligible contribution from the interlayer $\pi-\pi$ stacking of the ligands, which is responsible for the long-term photoconductivity of $\mathrm{Cu}$-THQ. These findings are unprecedented: the discovery of hot carriers with high mobility in Cu-THQ suggests the importance of design systems that can dissociate these highly delocalized hot carriers before they cool down. The revelation of the presence of longlived mobile carriers which are transported through the $\mathrm{O}-\mathrm{Cu}-\mathrm{O}$ motif in $\mathrm{CU}-\mathrm{THQ}$ implies not only the possibility of the further optimization of these materials through tuning the chain structure but also a great promise of the extraction of these long-lived excitons for effective use in photocatalysis, solar cells, and photo-electronics.

\section{Supporting Information}

The Supporting Information is available free of charge at https://pubs.acs.org/doi/10.1021/jacs.0c09000.

- Diffuse reflectance spectrum, $\mathrm{N}_{2}$ sorption isotherms, OTA spectra, OPTP kinetics, band structure calculation, EDX spectra, and tables for fitting parameters (PDF)

\section{Terms \& Conditions}

Most electronic Supporting Information files are available without a subscription to ACS Web Editions. Such files may be downloaded by article for research use (if there is a public use license linked to the relevant article, that license may permit other uses). Permission may be obtained from ACS for other uses through requests via the RightsLink permission system: http://pubs.acs.org/page/copyright/permissions.html.

\section{Author Contributions}

The manuscript was written through contributions of all authors. All authors have given approval to the final version of the manuscript.

\section{Notes}

The authors declare no competing financial interest.

C.A.S.: deceased July 26, 2020.

\section{Acknowledgments}

This research was supported by the U.S. Department of Energy, Office of Science, Office of Basic Energy Sciences, under Award No. DE-SC0020122. C.S. acknowledges support from the U.S. Department of Energy, Chemical Sciences, Geosciences, and Biosciences Division, Office of Basic Energy Sciences, Office of Science (Grant DEFG02-07ER15909) for the THz measurements. Use of the Advanced Photon Source at Argonne National Laboratory was supported by the U. S. Department of Energy, Office of Science, Office of Basic Energy Sciences, under Award No. DE-AC02-06CH11357. XPS investigations were performed the Irvine Materials Research Institute (IMRI) at the University of California, Irvine using instrumentation funded in part by the NSF Major Research Instrumentation Program under grant no. CHE-1338173. We specially thank Ich Tran at IMRI in 
assisting with XPS data collection. This work used the high-performance computing cluster at the Extreme Science and Engineering Discovery Environment (XSEDE) supported by NSF grant $\mathrm{ACl}-1053575$.

\section{References}

1 Janiak, C.; Vieth, J. K. MOFs, MILs and more: concepts, properties and applications for porous coordination networks (PCNs). New J. Chem. 2010, 34 (11), 2366- 2388, DOI: 10.1039/c0nj00275e

2 Muschielok, C.; Oberhofer, H. Aspects of semiconductivity in soft, porous metal-organic framework crystals. J. Chem. Phys. 2019, 151 (1), 015102, DOI: 10.1063/1.5108995

3 Sun, L.; Campbell, M. G.; Dinca, M. Electrically Conductive Porous Metal-Organic Frameworks. Angew. Chem., Int. Ed. 2016, 55 (11), 3566- 3579, DOI: 10.1002/anie.201506219

4 Yaghi, O. M.; O'Keeffe, M.; Ockwig, N. W.; Chae, H. K.; Eddaoudi, M.; Kim, J. Reticular synthesis and the design of new materials. Nature 2003, 423 (6941), 705- 714, DOI: 10.1038/nature01650

5 Latroche, M.; Surble, S.; Serre, C.; Mellot-Draznieks, C.; Llewellyn, P. L.; Lee, J. H.; Chang, J. S.; Jhung, S. H.; Ferey, G. Hydrogen storage in the giant-pore metal-organic frameworks MIL-100 and MIL101. Angew. Chem., Int. Ed. 2006, 45 (48), 8227-8231, DOI: 10.1002/anie. 200600105

6 Zhang, Z. J.; Zhao, Y. G.; Gong, Q. H.; Li, Z.; Li, J. MOFs for CO2 capture and separation from flue gas mixtures: the effect of multifunctional sites on their adsorption capacity and selectivity. Chem. Commun. 2013, 49 (7), 653-661, DOI: 10.1039/C2CC35561B

7 Dolgopolova, E. A.; Rice, A. M.; Martin, C. R.; Shustova, N. B. Photochemistry and photophysics of MOFs: steps towards MOF-based sensing enhancements. Chem. Soc. Rev. 2018, 47 (13), 4710- 4728, DOI: 10.1039/C7CS00861A

8 Zhou, Y. X.; Hu, W. H.; Yang, S. Z.; Zhang, Y. B.; Nyakuchena, J.; Duisenova, K.; Lee, S.; Fan, D. H.; Huang, J. SiteSelective Probes of Mixed-Node Metal Organic Frameworks for Photocatalytic Hydrogen Generation. J. Phys. Chem. C 2020, 124 (2), 1405-1412, DOI: 10.1021/acs.jpcc.9b09634

9 Furukawa, H.; Cordova, K. E.; O’Keeffe, M.; Yaghi, O. M. The chemistry and applications of metal-organic frameworks. Science 2013, 341 (6149), 1230444, DOI: 10.1126/science.1230444

10 Lazaro, I. A.; Forgan, R. S. Application of zirconium MOFs in drug delivery and biomedicine. Coord. Chem. Rev. 2019, 380, 230-259, DOI: 10.1016/j.ccr.2018.09.009

11 Kung, C.-W.; Han, P.-C.; Chuang, C.-H.; Wu, K. C.-W. Electronically conductive metal-organic framework-based materials. APL Mater. 2019, 7 (11), 110902, DOI: 10.1063/1.5125487

12 Li, P. F.; Wang, B. Recent Development and Application of Conductive MOFs. Isr. J. Chem. 2018, 58 (910), 1010- 1018, DOI: 10.1002/ijch.201800078

13 Xu, H. Q.; Yang, S. Z.; Ma, X.; Huang, J. E.; Jiang, H. L. Unveiling Charge-Separation Dynamics in CdS/MetalOrganic Framework Composites for Enhanced Photocatalysis. ACS Catal. 2018, 8 (12), 11615- 11621, DOI: 10.1021/acscatal.8b03233

14 Guo, F.; Yang, S. Z.; Liu, Y.; Wang, P.; Huang, J.; Sun, W. Y. Size Engineering of Metal-Organic Framework MIL101(Cr)-Ag Hybrids for Photocatalytic CO2 Reduction. ACS Catal. 2019, 9 (9), 8464-8470, DOI: 10.1021/acscatal.9b02126

15 Ko, M.; Mendecki, L.; Mirica, K. A. Conductive two-dimensional metal-organic frameworks as multifunctional materials. Chem. Commun. 2018, 54 (57), 7873- 7891, DOI: 10.1039/C8CC02871K

16 Sheberla, D.; Sun, L.; Blood-Forsythe, M. A.; Er, S.; Wade, C. R.; Brozek, C. K.; Aspuru-Guzik, A.; Dinca, M. High Electrical Conductivity in Ni-3(2,3,6,7,10,11-hexaiminotriphenylene)(2), a Semiconducting Metal-Organic Graphene Analogue. J. Am. Chem. Soc. 2014, 136 (25), 8859- 8862, DOI: 10.1021/ja502765n

17 Huang, X.; Sheng, P.; Tu, Z.; Zhang, F.; Wang, J.; Geng, H.; Zou, Y.; Di, C.-a.; Yi, Y.; Sun, Y. A two-dimensional $\pi-$ $\mathrm{d}$ conjugated coordination polymer with extremely high electrical conductivity and ambipolar transport behaviour. Nat. Commun. 2015, 6 (1), 1-8, DOI: 10.1038/ncomms8408

18 Qu, L. Y.; Iguchi, H.; Takaishi, S.; Habib, F.; Leong, C. F.; D’Alessandro, D. M.; Yoshida, T.; Abe, H.; Nishibori, E.; Yamashita, M. Porous Molecular Conductor: Electrochemical Fabrication of Through-Space Conduction Pathways among Linear Coordination Polymers. J. Am. Chem.

Soc. 2019, 141 (17), 6802-6806, DOI: 10.1021/jacs.9b01717 
19 Xie, L. S.; Alexandrov, E. V.; Skorupskii, G.; Proserpio, D. M.; Dinca, M. Diverse pi-pi stacking motifs modulate electrical conductivity in tetrathiafulvalene-based metal-organic frameworks. Chemical Science 2019, 10 (37), 8558-8565, DOI: 10.1039/C9SC03348C

20 Sun, L.; Miyakai, T.; Seki, S.; Dinca, M. Mn-2(2,5-disulfhydrylbenzene-1,4-dicarboxylate): A Microporous Metal-Organic Framework with Infinite (-Mn-S-)(infinity) Chains and High Intrinsic Charge Mobility. J. Am. Chem. Soc. 2013, 135 (22), 8185-8188, DOI: 10.1021/ja4037516

21 Dong, R.; Han, P.; Arora, H.; Ballabio, M.; Karakus, M.; Zhang, Z.; Shekhar, C.; Adler, P.; Petkov, P. S.; Erbe, A. High-mobility band-like charge transport in a semiconducting two-dimensional metal-organic framework. Nat. Mater. 2018, 17 (11), 1027- 1032, DOI: 10.1038/s41563-018-0189-z

22 Kung, C. W.; Goswami, S.; Hod, I.; Wang, T. C.; Duan, J. X.; Farha, O. K.; Hupp, J. T. Charge Transport in Zirconium-Based Metal-Organic Frameworks. Acc. Chem. Res. 2020, 53 (6), 1187-1195, DOI: 10.1021/acs.accounts.0c00106

23 Xie, L. S.; Skorupskii, G.; Dinca, M. Electrically Conductive Metal-Organic Frameworks. Chem. Rev. 2020, 120 (16), 8536- 8580, DOI: 10.1021/acs.chemrev.9b00766

24 Chen, D. S.; Xing, H. Z.; Su, Z. M.; Wang, C. G. Electrical conductivity and electroluminescence of a new anthracene-based metal-organic framework with pi-conjugated zigzag chains. Chem. Commun. 2016, 52 (10), 2019- 2022, DOI: 10.1039/C5CC09065B

25 Castaldelli, E.; Jayawardena, K. I.; Cox, D. C.; Clarkson, G. J.; Walton, R. I.; Le-Quang, L.; Chauvin, J.; Silva, S. R. P.; Demets, G. J.-F. Electrical semiconduction modulated by light in a cobalt and naphthalene diimide metal-organic framework. Nat. Commun. 2017, 8 (1), 1- 8, DOI: 10.1038/s41467-017-02215-7

26 Kamakura, Y.; Chinapang, P.; Masaoka, S.; Saeki, A.; Ogasawara, K.; Nishitani, S. R.; Yoshikawa, H.; Katayama, T.; Tamai, N.; Sugimoto, K. Semiconductive Nature of Lead-Based Metal-Organic Frameworks with Three-Dimensionally Extended Sulfur Secondary Building Units. J. Am. Chem. Soc. 2020, 142 (1), 27- 32, DOI: $10.1021 /$ jacs.9b10436

27 Allendorf, M. D.; Dong, R. H.; Feng, X. L.; Kaskel, S.; Matoga, D.; Stavila, V. Electronic Devices Using Open Framework Materials. Chem. Rev. 2020, 120 (16), 8581- 8640, DOI: 10.1021/acs.chemrev.0c00033

28 Sun, L.; Park, S. S.; Sheberla, D.; Dinca, M. Measuring and Reporting Electrical Conductivity in Metal Organic Frameworks: Cd-2(TTFTB) as a Case Study. J. Am. Chem. Soc. 2016, 138 (44), 14772-14782, DOI: 10.1021/jacs.6b09345

29 Pattengale, B.; SantaLucia, D. J.; Yang, S. Z.; Hu, W. H.; Liu, C. M.; Zhang, X. Y.; Berry, J. F.; Huang, J. Direct Observation of Node-to-Node Communication in Zeolitic Imidazolate Frameworks. J. Am. Chem. Soc. 2018, 140 (37), 11573- 11576, DOI: 10.1021/jacs.8b06727

30 Park, J.; Hinckley, A. C.; Huang, Z. H.; Feng, D. W.; Yakoyenko, A. A.; Lee, M.; Chen, S. C.; Zou, X.; Bao, Z. A. Synthetic Routes for a 2D Semiconductive Copper Hexahydroxybenzene Metal-Organic Framework. J. Am. Chem. Soc. 2018, 140 (44), 14533-14537, DOI: 10.1021/jacs.8b06666

31 Pattengale, B.; Neu, J.; Ostresh, S.; Hu, G. F.; Spies, J. A.; Okabe, R.; Brudvig, G. W.; Schmuttenmaer, C. A. Metal-Organic Framework Photoconductivity via Time-Resolved Terahertz Spectroscopy. J. Am. Chem. Soc. 2019, 141 (25), 9793-9797, DOI: 10.1021/jacs.9b04338

$32 \mathrm{Neu}$, J.; Regan, K. P.; Swierk, J. R.; Schmuttenmaer, C. A. Applicability of the thin-film approximation in terahertz photoconductivity measurements. Appl. Phys. Lett. 2018, 113 (23), 233901, DOI: 10.1063/1.5052232

33 Capobianco, M. D.; Pattengale, B.; Neu, J.; Schmuttenmaer, C. A. Single Copper Atoms Enhance Photoconductivity in g-C3N4. J. Phys. Chem. Lett. 2020, 11, 8873-8879, DOI: 10.1021/acs.jpclett.0c02756

$34 \mathrm{Neu}$, J.; Schmuttenmaer, C. A. Tutorial: An introduction to terahertz time domain spectroscopy (THz-TDS). J. Appl. Phys. 2018, 124 (23), 231101, DOI: 10.1063/1.5047659

35 Mortensen, J. J.; Hansen, L. B.; Jacobsen, K. W. Real-Space Grid Implementation of the Projector Augmented Wave Method. Phys. Rev. B: Condens. Matter Mater. Phys. 2005, 71 (3), 35109, DOI: 10.1103/PhysRevB.71.035109 
36 Enkovaara, J.; Rostgaard, C.; Mortensen, J. J.; Chen, J.; Dułak, M.; Ferrighi, L.; Gavnholt, J.; Glinsvad, C.; Haikola, V.; Hansen, H. A.; Kristoffersen, H. H.; Kuisma, M.; Larsen, A. H.; Lehtovaara, L.; Ljungberg, M.; Lopez-Acevedo, O.; Moses, P. G.; Ojanen, J.; Olsen, T.; Petzold, V.; Romero, N. A.; Stausholm-Møller, J.; Strange, M.; Tritsaris, G. A.; Vanin, M.; Walter, M.; Hammer, B.; Hakkinen, H.; Madsen, G. K. H.; Nieminen, R. M.; Nørskov, J. K.; Puska, M.; Rantala, T. T.; Schiøtz, J.; Thygesen, K. S.; Jacobsen, K. W. Electronic Structure Calculations with GPAW: A Real-Space Implementation of the Projector Augmented-Wave Method. J. Phys.: Condens. Matter 2010, 22 (25), 253202, DOI: 10.1088/0953$8984 / 22 / 25 / 253202$

37 Hjorth Larsen, A.; Jørgen Mortensen, J.; Blomqvist, J.; Castelli, I. E.; Christensen, R.; Dułak, M.; Friis, J.; Groves, M. N.; Hammer, B.ør.; Hargus, C.; Hermes, E. D.; Jennings, P. C.; Bjerre Jensen, P.; Kermode, J.; Kitchin, J. R.; Leonhard Kolsbjerg, E.; Kubal, J.; Kaasbjerg, K.; Lysgaard, S.; Bergmann Maronsson, J.; Maxson, T.; Olsen, T.; Pastewka, L.; Peterson, A.; Rostgaard, C.; Schiøtz, J.; Schutt, O.; Strange, M.; Thygesen, K. S.; Vegge, T.; Vilhelmsen, L.; Walter, M.; Zeng, Z.; Jacobsen, K. W. The Atomic Simulation Environment -a Python Library for Working with Atoms. J. Phys.: Condens. Matter 2017, 29 (27), 273002, DOI: 10.1088/1361-648X/aa680e

38 Perdew, J. P.; Ruzsinszky, A.; Csonka, G. I.; Vydrov, O. A.; Scuseria, G. E.; Constantin, L. A.; Zhou, X.; Burke, K. Restoring the Density-Gradient Expansion for Exchange in Solids and Surfaces. Phys. Rev. Lett. 2008, 100 (13), 136406, DOI: 10.1103/PhysRevLett.100.136406

39 Zhang, Y.; Yang, W. Comment on "Generalized Gradient Approximation Made Simple". Phys. Rev. Lett. 1998, 80 (4), 890, DOI: 10.1103/PhysRevLett.80.890

40 Setyawan, W.; Curtarolo, S. High-throughput electronic band structure calculations: Challenges and tools. Comput. Mater. Sci. 2010, 49 (2), 299-312, DOI: 10.1016/j.commatsci.2010.05.010

41 Kumar, S.; Yadav, M. Ultraviolet Absorption Spectra, Solvent Effect and Non-Linear Optical Properties of Tetrahydroxy-1, 4-quinone Hydrate by Hartee-Fock and Density Functional Theory. Asian J. Chem. 2017, 29 (10), 2241-2248, DOI: 10.14233/ajchem.2017.20741

42 Zhang, R.; Liu, J.; Gao, Y. F.; Hua, M. Q.; Xia, B. W.; Knecht, P.; Papageorgiou, A. C.; Reichert, J.; Barth, J. V.; Xu, H.; Huang, L.; Lin, N. On-surface Synthesis of a Semiconducting 2D Metal-Organic Framework Cu3(C6O6) Exhibiting Dispersive Electronic Bands. Angew. Chem., Int. Ed. 2020, 59 (7), 2669-2673, DOI: 10.1002/anie.201913698

43 Huang, J.; Mulfort, K. L.; Du, P. W.; Chen, L. X. Photodriven Charge Separation Dynamics in CdSe/ZnS Core/Shell Quantum Dot/Cobaloxime Hybrid for Efficient Hydrogen Production. J. Am. Chem. Soc. 2012, 134 (40), 16472-16475, DOI: 10.1021/ja3062584

44 Zhou, Y.; Yang, S.; Huang, J. Light-driven hydrogen production from aqueous solutions based on a new Dubois-type nickel catalyst. Phys. Chem. Chem. Phys. 2017, 19 (11), 7471-7475, DOI: 10.1039/C7CP00247E

45 Yu, J. R.; Park, J.; Van Wyk, A.; Rumbles, G.; Deria, P. Excited-State Electronic Properties in Zr-Based MetalOrganic Frameworks as a Function of a Topological Network. J. Am. Chem. Soc. 2018, 140 (33), 10488- 10496, DOI: 10.1021/jacs.8b04980

46 Borfecchia, E.; Garino, C.; Salassa, L.; Lamberti, C. Synchrotron ultrafast techniques for photoactive transition metal complexes. Philos. Trans. R. Soc., A 2013, 371 (1995), 20120132, DOI: 10.1098/rsta.2012.0132

47 DeBeer, S.; Randall, D. W.; Nersissian, A. M.; Valentine, J. S.; Hedman, B.; Hodgson, K. O.; Solomon, E. I. X-ray absorption edge and EXAFS studies of the blue copper site in stellacyanin: Effects of axial amide coordination. J. Phys. Chem. B 2000, 104 (46), 10814-10819, DOI: 10.1021/jp001334d

48 Choy, J. H.; Kim, D. K.; Park, J. C.; Choi, S. N.; Kim, Y. J. Intracrystalline and electronic structures of copper(II) complexes stabilized in two-dimensional aluminosilicate. Inorg. Chem. 1997, 36 (2), 189- 195, DOI: 10.1021/ic960631n

49 Moonshiram, D.; Garrido-Barros, P.; Gimbert-Surinach, C.; Picon, A.; Liu, C. M.; Zhang, X. Y.; Karnahl, M.; Llobet, A. Elucidating the Nature of the Excited State of a Heteroleptic Copper Photosensitizer by using Time-Resolved X-ray Absorption Spectroscopy. Chem. - Eur. J. 2018, 24 (24), 6464- 6472, DOI: 10.1002/chem.201800330 
50 DuBois, J. L.; Mukherjee, P.; Stack, T. D. P.; Hedman, B.; Solomon, E. I.; Hodgson, K. O. A systematic K-edge Xray absorption spectroscopic study of $\mathrm{Cu}(\mathrm{III})$ sites. J. Am. Chem. Soc. 2000, 122 (24), 5775- 5787, DOI: 10.1021/ja993134p

51 Beard, M. C.; Turner, G. M.; Schmuttenmaer, C. A. Transient photoconductivity in GaAs as measured by timeresolved terahertz spectroscopy. Phys. Rev. B: Condens. Matter Mater. Phys. 2000, 62 (23), 15764-15777, DOI: 10.1103/PhysRevB.62.15764

52 Nemes, C. T.; Koenigsmann, C.; Schmuttenmaer, C. A. Functioning Photoelectrochemical Devices Studied with Time-Resolved Terahertz Spectroscopy. J. Phys. Chem. Lett. 2015, 6 (16), 3257- 3262, DOI: 10.1021/acs.jpclett.5b01473

53 Alberding, B. G.; Heilweil, E. J. In Time-resolved terahertz spectroscopy of electrically conductive metal-organic frameworks doped with redox active species, Organic Photovoltaics XVI, International Society for Optics and Photonics: 2015; p 95671L.

54 Pattengale, B.; Freeze, J. G.; Guberman-Pfeffer, M. J.; Okabe, R.; Ostresh, S.; Chaudhuri, S.; Batista, V. S.; Schmuttenmaer, C. A. A conductive metal-organic framework photoanode. Chemical Science 2020, 11 (35), 9593-9603, DOI: 10.1039/DOSC04302H

55 Spies, J. A.; Neu, J.; Tayvah, U. T.; Capobianco, M. D.; Pattengale, B.; Ostresh, S.; Schmuttenmaer, C. A. Terahertz Spectroscopy of Emerging Materials. J. Phys. Chem. C 2020, 124 (41), 22335- 22346, DOI: 10.1021/acs.jpcc.0c06344

56 Neu, J.; Ostresh, S.; Regan, K. P.; Spies, J. A.; Schmuttenmaer, C. A. Influence of Dye Sensitizers on Charge Dynamics in SnO2 Nanoparticles Probed with THz Spectroscopy. J. Phys. Chem. C 2020, 124 (6), 3482-3488, DOI: 10.1021/acs.jpcc.9b11024

57 Regan, K. P.; Swierk, J. R.; Neu, J.; Schmuttenmaer, C. A. Frequency-Dependent Terahertz Transient Photoconductivity of MesoporoUs SnO2 Films. J. Phys. Chem. C 2017, 121 (29), 15949- 15956, DOI: 10.1021/acs.jpcc.7b02318 